\title{
Updated search for long-lived particles decaying to jet pairs
}

\author{
LHCb Collaboration ${ }^{\star}$ \\ CERN, 1211 Geneva 23, Switzerland
}

Received: 23 May 2017 / Accepted: 31 August 2017 / Published online: 29 November 2017

(C) CERN for the benefit of the LHCb collaboration 2017. This article is an open access publication

\begin{abstract}
A search is presented for long-lived particles with a mass between 25 and $50 \mathrm{GeV} / c^{2}$ and a lifetime between 2 and 500 ps, using proton-proton collision data corresponding to an integrated luminosity of $2.0 \mathrm{fb}^{-1}$, collected by the LHCb detector at centre-of-mass energies of 7 and $8 \mathrm{TeV}$. The particles are assumed to be pair-produced in the decay of a $125 \mathrm{GeV} / c^{2}$ Standard-Model-like Higgs boson. The experimental signature is a single long-lived particle, identified by a displaced vertex with two associated jets. No excess above background is observed and limits are set on the production cross-section as a function of the mass and lifetime of the long-lived particle.
\end{abstract}

\section{Introduction}

Various extensions of the Standard Model (SM) feature new particles whose couplings to lighter states are sufficiently small to result in detectable lifetimes. In this paper we report on a search for such long-lived particles, which are assumed to be pair-produced in the decay of a Standard-Model-like Higgs boson, and subsequently decay into a quark-antiquark pair. Such a signature is present in models with a hiddensector non-Abelian gauge group, where the Standard Model Higgs boson acts as a portal [1-5]. The new scalar particle represents the lightest state in the hidden sector and is called a hidden-valley pion $\left(\pi_{\mathrm{v}}\right)$ throughout this paper. Experimental constraints on the properties of the Higgs boson of mass $125 \mathrm{GeV} / c^{2}$ observed by the ATLAS and CMS collaborations $[6,7]$ still allow for branching fractions of non-SM decay modes of up to $30 \%$ [8].

Data collected with the LHCb experiment in 2011 and 2012 are used for this analysis, restricted to periods in which suitable triggers were available. The data sample analysed corresponds to $0.62 \mathrm{fb}^{-1}$ at a centre-of-mass energy of $\sqrt{s}=7 \mathrm{TeV}$ and $1.38 \mathrm{fb}^{-1}$ at $\sqrt{s}=8 \mathrm{TeV}$. In simulated events with $\pi_{\mathrm{v}}$ pairs originating from a Higgs boson decay it

^e-mail: pieter.david@cern.ch is found that in most cases no more than one of the two $\pi_{\mathrm{v}}$ decays occurs inside the LHCb acceptance. Consequently, the experimental signature is a single $\pi_{v}$ particle. The candidate is identified by its decay to two hadronic jets originating from a displaced vertex, with a transverse distance to the proton-proton collision axis $\left(R_{x y}\right)$ of at least $0.4 \mathrm{~mm}$. The vertex is required to have at least five tracks reconstructed in the $\mathrm{LHCb}$ vertex detector. The analysis is sensitive to $\pi_{\mathrm{v}}$ particles with a mass between 25 and $50 \mathrm{GeV} / c^{2}$ and a lifetime between 2 and $500 \mathrm{ps}$. The lifetime range is limited due to the presence of large prompt backgrounds at short decay times and the acceptance of the vertex detector for long decay times. The lower boundary on the mass range arises from the requirement to identify two hadronic jets while the upper boundary is driven by the geometric acceptance of the detector.

This paper presents an update of an earlier analysis, which considered only the data set corresponding to an integrated luminosity of $0.62 \mathrm{fb}^{-1}$ collected at $\sqrt{s}=7 \mathrm{TeV}$ [9]. Similar searches for hidden-valley particles decaying to jet pairs were performed by the D0 [10], CDF [11], ATLAS [12-14] and CMS [15] collaborations. Compared to these analyses, this search is sensitive to $\pi_{\mathrm{v}}$ particles with relatively low mass and lifetime. The LHCb collaboration has also performed a search for events with two displaced high-multiplicity vertices [16] and a search for events with a lepton from a highmultiplicity displaced vertex [17] in the context of SUSY models, and several searches for so far unknown long-lived particles in $B$-meson decays [18-21].

\section{Detector and event simulation}

The LHCb detector [22,23] is a single-arm forward spectrometer covering the pseudorapidity range $2<\eta<5$, designed for the study of particles containing $b$ or $c$ quarks. The detector includes a high-precision tracking system consisting of a silicon-strip vertex detector (VELO) surrounding the $p p$ interaction region, a large-area silicon-strip detec- 
tor located upstream of a dipole magnet with a bending power of about $4 \mathrm{Tm}$, and three stations of silicon-strip detectors and straw drift tubes placed downstream of the magnet. The tracking system provides a measurement of the momentum, $p$, of charged particles with a relative uncertainty that varies from $0.5 \%$ at low momentum to $1.0 \%$ at $200 \mathrm{GeV} / c$. The minimum distance of a track to a primary vertex (PV), the impact parameter (IP), is measured with a resolution of $\left(15+(29 \mathrm{GeV} / c) / p_{\mathrm{T}}\right) \mu \mathrm{m}$, where $p_{\mathrm{T}}$ is the component of the momentum transverse to the collision axis. Different types of charged hadrons are distinguished using information from two ring-imaging Cherenkov detectors. Photons, electrons and hadrons are identified by a calorimeter system consisting of scintillating-pad (SPD) and preshower detectors, an electromagnetic calorimeter and a hadronic calorimeter. Muons are identified by a system composed of alternating layers of iron and multiwire proportional chambers.

The model for the production of $\pi_{\mathrm{v}}$ particles through the Higgs portal is fully specified by three parameters: the mass of the Higgs boson and the mass and lifetime of the $\pi_{\mathrm{v}}$. The Higgs boson mass is taken to be $125 \mathrm{GeV} / c^{2}$, and its production through the gluon-gluon fusion process is simulated with the PYTHIA8 generator [24], with a specific LHCb configuration [25] and using the CTEQ6 leading-order set of parton density functions [26]. The interaction of the generated particles with the detector, and its response, are implemented using the GEANT4 toolkit [27,28] as described in Ref. [29]. Signal samples with $\pi_{\mathrm{v}}$ masses of $25,35,43$ and $50 \mathrm{GeV} / c^{2}$ and lifetimes of 10 and 100 ps are generated. In the simulated events the long-lived particles decay exclusively as $\pi_{\mathrm{v}} \rightarrow b \bar{b}$, since this decay mode is generally preferred in the Higgs portal model. Samples with decays to $c$ - and $s$-quark pairs are generated as well, but only in the scenario with a mass of $35 \mathrm{GeV} / \mathrm{c}^{2}$ and a lifetime of $10 \mathrm{ps}$.

\section{Event selection}

The experimental signature for this analysis is a single displaced vertex with two associated jets. Only decays that produce a sufficient number of tracks in the VELO for a vertex to be reconstructed are considered. Due to the geometry of the vertex detector, this restricts the sample to decay points up to about $200 \mathrm{~mm}$ from the nominal interaction point along the beam direction, and up to about $30 \mathrm{~mm}$ in the transverse direction, thereby limiting the decay time acceptance. The selection strategy is the same as used in the analysis of Ref. [9]. Reconstructed tracks are used to find the decay vertex, and jets are built out of reconstructed particles compatible with originating from that vertex. Constraints on the signal yield are determined from a fit to the dijet invariant mass distribution. The main source of background is displaced vertices from heavy-flavour decays or interactions of particles with detector material. To take into account the strong dependence of the background level on the separation from the beam axis, different selection criteria are used in different bins of $R_{x y}$, and the final fit is performed in bins of this variable.

The selection consists of online (trigger) and offline parts. The trigger [30] is divided into a hardware (L0) and a software (HLT) stage. The L0 requires a muon with high $p_{\mathrm{T}}$ or a hadron, photon or electron with high transverse energy in the calorimeters. In order to reduce the processing time of the subsequent trigger stages, events with a large hit multiplicity in the SPD are discarded. The software stage is divided into two parts, which for this analysis differ between the 2011 and 2012 data. In the 2011 sample, the first software stage (HLT1) requires a single high- $p_{\mathrm{T}}$ track with a large impact parameter. The HLT1 selection for the 2012 sample was complemented with a two-track vertex signature with looser track quality criteria, in order to improve the efficiency at large displacements. At the second stage of the software trigger (HLT2), events are required to pass either a dedicated inclusive displaced-vertex selection or a standard topological $B$ decay selection, which requires a two-, three- or four-track vertex with a significant displacement from all PVs [30]. The inclusive displaced-vertex selection uses an algorithm similar to that used for the LHCb primary vertex reconstruction [31]. A combination of requirements on the minimum number of tracks in the vertex (at least four), the distance $R_{x y}$ of the vertex to the beam axis (at least $0.4 \mathrm{~mm}$ ), the invariant mass of the particles associated with the vertex (at least $2 \mathrm{GeV} / c^{2}$ ) and the scalar sum $p_{\mathrm{T}}$ of the tracks that form the vertex (at least $3 \mathrm{GeV} / c$ ), is used to define a set of trigger selections with sufficiently low rate.

Before the offline selection can be applied, the displaced vertex corresponding to the decay of the $\pi_{\mathrm{v}}$ candidate must be reconstructed. For those events in which the HLT2 inclusive displaced-vertex selection was successful, the same vertex candidate found in the trigger is used; this approach differs from that used in the previous LHCb analysis [9] and simplifies the evaluation of systematic uncertainties. For events selected only by the topological $B$ trigger, a modified version of the algorithm is run on the output of the offline reconstruction with the following criteria: vertices with $0.4<R_{x y}<1 \mathrm{~mm}$ must have at least eight tracks and the invariant mass of the system must exceed $10 \mathrm{GeV} / c^{2}$, vertices with $1<R_{x y}<5 \mathrm{~mm}$ must have at least six tracks, and those with $R_{x y}>5 \mathrm{~mm}$ must have at least five tracks. To exclude background due to interactions with the detector material, vertices inside a veto region around the VELO detector elements are discarded. Events with many parallel displaced tracks, which can arise from machine background, are identified by the azimuthal distribution of hits in the VELO and are also discarded.

Next, jets are reconstructed following a particle flow approach. The same set of inputs as in Ref. [32] is used, 
namely tracks of charged particles and calorimeter energy deposits, after subtraction of the energy associated with charged particles. To remove background, tracks that are compatible with coming from a PV, tracks with a smaller impact parameter to any primary vertex than to the displaced vertex, and tracks that have an impact parameter to the displaced vertex larger than $2 \mathrm{~mm}$ are all discarded. The anti- $k_{T}$ jet clustering algorithm is used [33], with a distance parameter of $R=0.7$. The jet momentum and jet mass are calculated from the four-vectors of all constituents of the jet. In simulated events the jet energy response is found to be close to unity except for the lowest jet momenta, near the minimally required transverse momentum of $5 \mathrm{GeV} / c$. Therefore, no jet energy correction was applied for this search.

To enhance the jet purity the fraction of the jet energy carried by charged particles should be at least 0.1 , there should be at least one track with transverse momentum above $0.9 \mathrm{GeV} / c$, no pair of constituents should carry $90 \%$ of the jet energy, and no single charged or neutral constituent should contribute more than 70 or $50 \%$ of the total energy, respectively. To ensure that they can reliably be associated to a vertex, the jets are also required to have at least two constituents with track segments in the VELO. To account for differences in trigger and background conditions, for the 2012 data this requirement was tightened to at least four segments for $R_{x y}<1 \mathrm{~mm}$, and at least three segments for $1<R_{x y}<2 \mathrm{~mm}$. For each jet an origin point is reconstructed from the jet constituents with VELO information. The jet trajectory is defined based on this origin point and the momentum of the jet. Any jet whose trajectory does not point back to the candidate vertex within $2 \mathrm{~mm}$, or points more closely to a primary vertex, is removed. Only candidates with at least two jets passing these criteria are retained.

Two final criteria are applied to the dijet candidates. The first is that the momentum vector of the dijet candidate should be aligned with the displacement vector from a PV to the reconstructed vertex position. This is implemented as a requirement on the dijet invariant mass divided by the corrected mass, $m / m_{\text {corr }}>0.7$. The corrected mass is computed as $m_{\text {corr }}=\sqrt{m^{2}+(p \sin \theta)^{2}}+p \sin \theta$ [34], where $m$ and $p$ are the reconstructed mass and momentum of the dijet, and $\theta$ is the minimum angle between the momentum vector and the displacement vectors to the vertex from any $\mathrm{PV}$ in the event. A requirement on $m / m_{\text {corr }}$ is preferred over one on the angle $\theta$ itself, since its efficiency depends less strongly on the boost and the mass of the candidate [35]. The second criterion is that the kinematic separation of the jets should satisfy $\Delta R=\sqrt{(\Delta \eta)^{2}+(\Delta \phi)^{2}}<2.2$, where $\Delta \eta$ and $\Delta \phi$ are the pseudorapidity and azimuthal angle differences between the two jets, respectively. This reduces the tail in the dijet invariant mass distribution by suppressing the remaining back-to-back dijet background.
Table 1 Number of selected candidates per generated $H^{0} \rightarrow \pi_{\mathrm{v}} \pi_{\mathrm{v}}$ event (efficiency) in percent for different $\pi_{\mathrm{v}} \rightarrow q \bar{q}, q=b, c, s$ models for 2011 and 2012 data taking conditions, as derived from simulation. The relative statistical uncertainty on the efficiency due to the limited size of the simulated sample is less than a few percent

\begin{tabular}{lllllll}
\hline & $\pi_{\mathrm{v}} \operatorname{mass}\left(\mathrm{GeV} / c^{2}\right)$ & 2011 & & & 2012 & \\
& & $10 \mathrm{ps}$ & $100 \mathrm{ps}$ & & $10 \mathrm{ps}$ & $100 \mathrm{ps}$ \\
\hline$\pi_{\mathrm{v}} \rightarrow b \bar{b}$ & 25 & 0.45 & 0.097 & 0.46 & 0.111 \\
$\pi_{\mathrm{v}} \rightarrow b \bar{b}$ & 35 & 0.80 & 0.176 & 0.83 & 0.224 \\
$\pi_{\mathrm{v}} \rightarrow b \bar{b}$ & 43 & 0.73 & 0.190 & 0.77 & 0.222 \\
$\pi_{\mathrm{v}} \rightarrow b \bar{b}$ & 50 & 0.49 & 0.141 & 0.54 & 0.171 \\
$\pi_{\mathrm{v}} \rightarrow c \bar{c}$ & 35 & 1.35 & & 1.35 & \\
$\pi_{\mathrm{v}} \rightarrow s \bar{s}$ & 35 & 1.30 & & 1.19 & \\
\hline
\end{tabular}

The overall efficiency to reconstruct and select displaced $\pi_{\mathrm{v}}$ decays in the simulated samples is summarized in Table 1 for the 2011 and 2012 data taking conditions. A large part of the inefficiency is due to the detector acceptance, which is about $13 \%(8 \%)$ and $6.5 \%(5.5 \%)$ for $\pi_{\mathrm{v}}$ particles with a lifetime of $10 \mathrm{ps}(100 \mathrm{ps})$ and masses of 25 and $50 \mathrm{GeV} / \mathrm{c}^{2}$, respectively. Other important contributions are due to the selection on the displacement from the beamline, requirements on the minimum number of tracks forming the vertex, the material interaction veto, the reduction in VELO tracking efficiency at large displacements, and the jet selection [36]. The efficiency for long-lived particles decaying to $s$ - and $c$ quark pairs is higher than for decays to $b$-quark pairs due to the larger number of tracks originating directly from the $\pi_{\mathrm{v}}$ decay vertex.

\section{Systematic uncertainties}

Systematic uncertainties on the efficiency are obtained from studies of data-simulation differences in control samples. They are reported in Tables 2 and 3, for the 2011 and 2012 conditions, respectively, and discussed in more detail below. Uncertainties on the signal efficiency due to parton-density distributions, the simulation of fragmentation and hadronization, and the Higgs boson production cross-section and kinematics are not taken into account.

The vertex reconstruction efficiency can be split into two parts, namely the track reconstruction efficiency and the vertex finding efficiency. The track reconstruction efficiency is described by the simulation to within a few percent, including for highly displaced and low-momentum tracks [37-39]. The effect of a systematic change in this efficiency is studied by randomly removing $2 \%$ of the signal tracks and reapplying all selection criteria.

The vertex finding algorithm is not fully efficient even if all tracks are reconstructed. In particular, the efficiency to 
Table 2 Overview of the contributions to the relative systematic uncertainty on the signal efficiency and luminosity (in percent) for different signal samples in 2011 conditions. The uncertainty on the total efficiency is obtained by summing the individual contributions in quadrature
Table 3 Overview of the contributions to the relative systematic uncertainty on the signal efficiency and luminosity (in percent) for different signal samples in 2012 conditions. The uncertainty on the total efficiency is obtained by summing the individual contributions in quadrature

\begin{tabular}{|c|c|c|c|c|c|c|c|c|c|c|}
\hline \multirow{2}{*}{$\begin{array}{l}\pi_{\mathrm{v}} \text { mass }\left(\mathrm{GeV} / c^{2}\right) \\
\pi_{\mathrm{v}} \text { lifetime }(\mathrm{ps})\end{array}$} & \multicolumn{2}{|l|}{25} & \multicolumn{2}{|l|}{35} & \multicolumn{2}{|l|}{43} & \multicolumn{2}{|l|}{50} & \multirow{2}{*}{$\frac{35, c \bar{c}}{10}$} & \multirow{2}{*}{$\frac{35, s \bar{s}}{10}$} \\
\hline & 10 & 100 & 10 & 100 & 10 & 100 & 10 & 100 & & \\
\hline Tracking efficiency & 4.2 & 4.1 & 3.3 & 3.2 & 3.0 & 2.8 & 3.0 & 2.7 & 1.8 & 1.7 \\
\hline Vertex finding & 3.8 & 4.2 & 3.3 & 3.9 & 2.8 & 3.7 & 3.7 & 2.6 & 2.9 & 2.8 \\
\hline Jet reconstruction & 3.1 & 3.1 & 1.6 & 1.6 & 0.7 & 0.7 & 0.5 & 0.5 & 0.9 & 1.0 \\
\hline Jet identification & 3.0 & 3.0 & 3.0 & 3.0 & 3.0 & 3.0 & 3.0 & 3.0 & 3.0 & 3.0 \\
\hline Jet direction & 7.0 & 7.0 & 6.0 & 6.0 & 7.4 & 7.4 & 8.5 & 8.5 & 5.9 & 5.7 \\
\hline L0 & 4.0 & 4.0 & 3.0 & 3.0 & 3.0 & 3.0 & 2.0 & 2.0 & 1.8 & 2.1 \\
\hline$N_{\text {SPD }}$ & 1.7 & 1.7 & 2.0 & 2.0 & 1.6 & 1.6 & 2.3 & 2.3 & 1.7 & 1.6 \\
\hline HLT1 & 2.0 & 2.0 & 2.0 & 2.0 & 2.0 & 2.0 & 2.0 & 2.0 & 2.0 & 2.0 \\
\hline HLT2 & 3.0 & 3.0 & 3.0 & 3.0 & 3.0 & 3.0 & 3.0 & 3.0 & 3.0 & 3.0 \\
\hline Total efficiency & 11.5 & 11.6 & 9.8 & 10.0 & 10.3 & 10.5 & 11.2 & 10.9 & 8.7 & 8.6 \\
\hline Luminosity & 1.7 & 1.7 & 1.7 & 1.7 & 1.7 & 1.7 & 1.7 & 1.7 & 1.7 & 1.7 \\
\hline
\end{tabular}

\begin{tabular}{|c|c|c|c|c|c|c|c|c|c|c|}
\hline \multirow{2}{*}{$\begin{array}{l}\pi_{\mathrm{v}} \text { mass }\left(\mathrm{GeV} / c^{2}\right) \\
\pi_{\mathrm{v}} \text { lifetime }(\mathrm{ps})\end{array}$} & \multicolumn{2}{|l|}{25} & \multicolumn{2}{|l|}{35} & \multicolumn{2}{|l|}{43} & \multicolumn{2}{|l|}{50} & \multirow{2}{*}{$\frac{35, c \bar{c}}{10}$} & \multirow{2}{*}{$\begin{array}{l}35, s \bar{s} \\
10\end{array}$} \\
\hline & 10 & 100 & 10 & 100 & 10 & 100 & 10 & 100 & & \\
\hline Tracking efficiency & 3.1 & 2.8 & 2.4 & 2.4 & 2.2 & 2.1 & 2.0 & 1.7 & 1.2 & 1.1 \\
\hline Vertex finding & 4.2 & 4.5 & 3.8 & 4.4 & 3.4 & 4.1 & 3.1 & 3.9 & 3.4 & 3.5 \\
\hline Jet reconstruction & 2.7 & 2.7 & 1.1 & 1.1 & 0.7 & 0.7 & 0.3 & 0.3 & 0.9 & 1.0 \\
\hline Jet identification & 3.0 & 3.0 & 3.0 & 3.0 & 3.0 & 3.0 & 3.0 & 3.0 & 3.0 & 3.0 \\
\hline Jet direction & 5.8 & 5.8 & 5.3 & 5.3 & 6.1 & 6.1 & 7.9 & 7.9 & 5.3 & 5.8 \\
\hline L0 & 4.0 & 4.0 & 2.5 & 2.5 & 2.0 & 2.0 & 2.0 & 2.0 & 2.0 & 2.0 \\
\hline$N_{\text {SPD }}$ & 2.2 & 2.2 & 2.5 & 2.5 & 2.5 & 2.5 & 2.5 & 2.5 & 2.4 & 2.1 \\
\hline HLT1 & 2.0 & 2.0 & 2.0 & 2.0 & 2.0 & 2.0 & 2.0 & 2.0 & 2.0 & 2.0 \\
\hline HLT2 & 3.0 & 3.0 & 3.0 & 3.0 & 3.0 & 3.0 & 3.0 & 3.0 & 3.0 & 3.0 \\
\hline Total efficiency & 10.5 & 10.6 & 9.2 & 9.4 & 9.1 & 9.5 & 10.4 & 10.6 & 8.6 & 8.9 \\
\hline Luminosity & 1.2 & 1.2 & 1.2 & 1.2 & 1.2 & 1.2 & 1.2 & 1.2 & 1.2 & 1.2 \\
\hline
\end{tabular}

find a low-multiplicity secondary vertex is reduced in the proximity of a high-multiplicity PV. The effect is studied in data and simulation using exclusively reconstructed $B^{0} \rightarrow$ $J / \Psi K^{* 0}$ decays, which can be selected with high purity without tight requirements on the vertex. The efficiency for the displaced vertex reconstruction algorithm to find the $B^{0}$ candidate is measured as a function of the displacement $R_{x y}$ in data and simulation [36]. The difference, weighted by the $R_{x y}$ distribution of the signal candidates, is used to derive a systematic uncertainty.

Systematic uncertainties related to the jet reconstruction can be introduced in two ways: through differences between data and simulation in the jet reconstruction efficiency and through differences between data and simulation in the resolution on the jet energy and direction, which enter the dijet candidate kinematic and $m / m_{\text {corr }}$ selection and the dijet invariant mass shape. The jet reconstruction efficiency has been studied previously in measurements of the $Z+$ jet and $Z+b$-jet cross-sections and was found to be consistent between data and simulation $[32,40]$. The $Z \rightarrow \mu^{+} \mu^{-}+$jet sample is used to study jet-related systematic effects for this analysis as well. To mimic the selection of the particle-flow inputs, the PV associated to the $Z$ is used as a proxy for the displaced vertex.

The difference between data and simulation with the largest impact on the jet reconstruction efficiency is the energy response to low- $p_{\mathrm{T}}$ jets, close to the threshold of $5 \mathrm{GeV} / c$. The sensitivity to a different energy response in data and simulation is evaluated by increasing the minimum jet $p_{\mathrm{T}}$ for candidates passing the full offline selection by $10 \%$, which is the uncertainty on the jet energy scale. The change in the overall selection efficiency is assigned as a systematic uncertainty. By replacing the jet identification criteria with a requirement on the $p_{\mathrm{T}}$ balance between the leading jet and the $Z$ boson, the $Z \rightarrow \mu^{+} \mu^{-}$sample can also be used to study the difference in jet identification efficiency between data and simulation. No difference larger than $3 \%$ relative is seen, which is assigned as a systematic uncertainty.

To validate the simulation of the jet-direction resolution the jet-direction is estimated separately with the charged and 
Fig. 1 Dijet invariant mass distribution in the different $R_{x y}$ bins, for the 2011 data sample. For illustration, the best fit with a signal $\pi_{\mathrm{v}}$ model with mass $35 \mathrm{GeV} / c^{2}$ and lifetime $10 \mathrm{ps}$ is overlaid. The solid blue line indicates the total background model, the short-dashed green line indicates the signal model for signal strength $\mu=1$, and the long-dashed red line indicates the best-fit signal strength
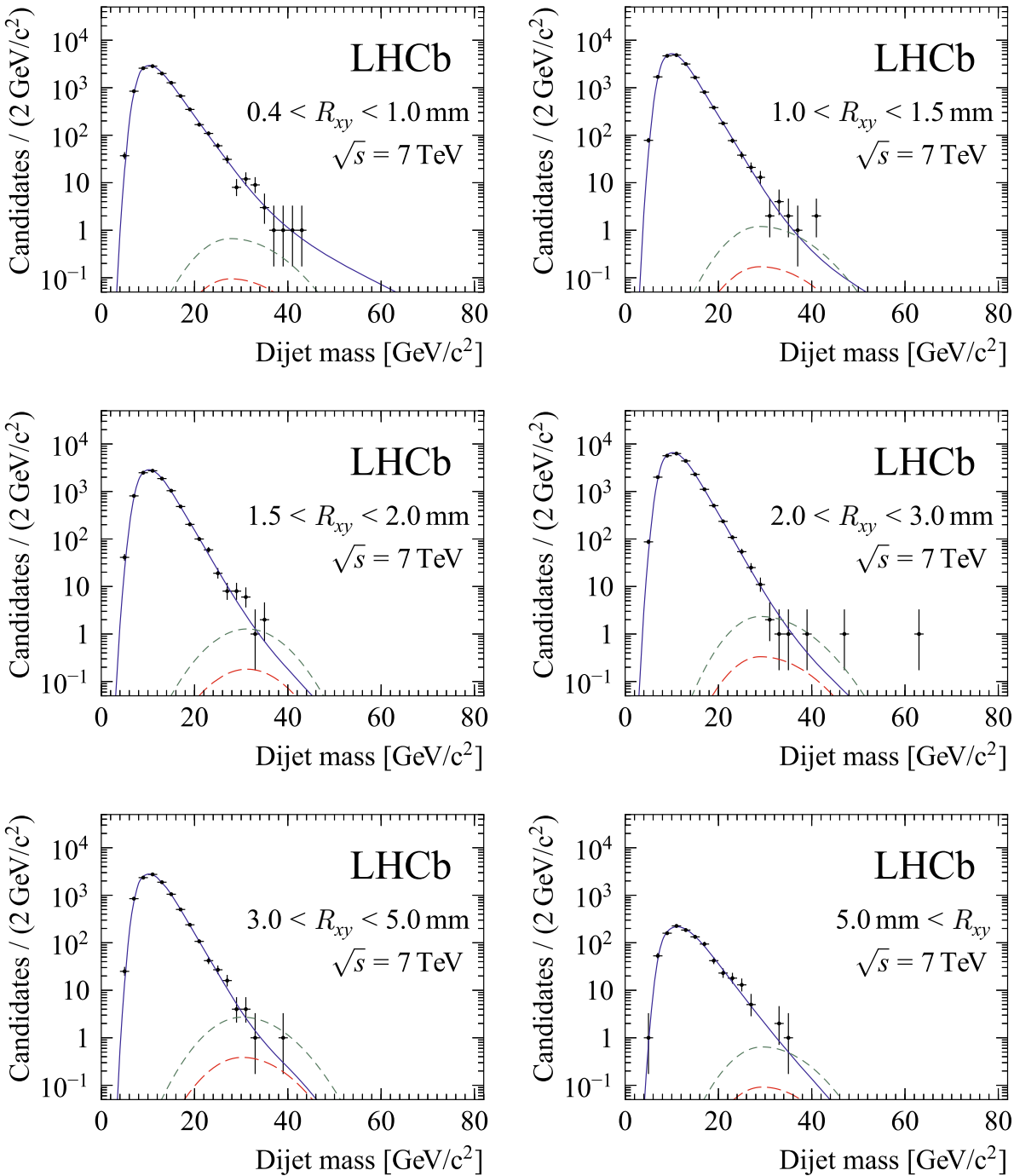

neutral components of the jet in $Z+$ jet events. The distribution of the charged-neutral difference in the estimated direction is found to be consistent between data and simulation for both the $\eta$ and the $\phi$ projection, and across the full range of $p_{\mathrm{T}}$. To quantify the effect on the $\pi_{\mathrm{V}}$ signal efficiency, an additional smearing to the jet-direction is applied to jets of selected candidates in the simulation. The jet angles with respect to the beam direction are smeared independently in the horizontal and vertical planes by about one third of the resolution, which is the largest value compatible with the comparison of data and simulation in $Z+$ jet events.

The systematic uncertainty related to the L0 trigger selection consists of two parts, due to differences in the L0 calorimeter trigger response between data and simulation, and due to the difference between data and simulation in the distribution of the SPD hit multiplicity $N_{\text {SPD }}$. The first is evaluated by studying the LO calorimeter trigger response on jets reconstructed in $Z+$ jet events, where the trigger decision is made based on the $Z \rightarrow \mu^{+} \mu^{-}$decay products, and is independent of the jet. The observed data-simulation differences are propagated to the $\pi_{\mathrm{v}}$ reconstruction efficiency and correspond to systematic uncertainties of $2-4 \%$, depending on the $\pi_{\mathrm{v}}$ mass. Jets in $Z+$ jet events are mostly light-quark jets, while our benchmark signal decays to $b$ quarks. It is found in simulated events that the efficiency of the LO calorimeter trigger is practically independent of jet flavour. A small fraction of $b$-quark jets is triggered exclusively by the L0 muon trigger, which is well modelled in the simulation.

The second part of the L0 systematic uncertainty arises because the SPD multiplicity is not well described in the simulation. This effect is studied with a $Z \rightarrow \mu^{+} \mu^{-}$sample triggered by the dimuon L0 selection, which applies only a loose selection on this quantity. An efficiency correction is derived, which is about $90 \%$ for 2011 data, and about $85 \%$ for 2012 data, with an uncertainty of $2-3 \%$. The difference in the correction between the different $\pi_{\mathrm{v}}$ models is smaller 
than the systematic variation. This correction is applied to the overall detection efficiency derived from the simulation and the uncertainty is taken as a systematic uncertainty.

The differences between data and simulation in the HLT1 selection are dominated by the track reconstruction efficiency, which was discussed above, and additional track quality criteria. One such difference is due to a requirement on the number of VELO hits for displaced tracks. It is characterized using $B^{0} \rightarrow J / \Psi K^{* 0}$ decays selected with triggers that do not apply such a requirement. For this sample the selection efficiency was found to be $2 \%$ higher in data than in simulated events, which is assigned as a systematic uncertainty. For $\pi_{\mathrm{v}}$ decays the final-state track multiplicity is larger, which dilutes effects due to a mismodelling of the single-track efficiency.

The main source of systematic uncertainty in the HLT2 selection is the vertex reconstruction efficiency, which was discussed above. The efficiency of the topological $B$ trigger, which is relevant for a subset of the candidates, is accurately described in simulation. It is measured as a function of $R_{x y}$ in data and simulation using $B^{0} \rightarrow J / \Psi K^{* 0}$ candidates that are selected by a different, dimuon-based, trigger criterion. A maximum difference of $2-3 \%$ is observed, which is assigned as a systematic uncertainty.

\section{Results}

Constraints on the presence of a signal are derived from a fit to the dijet invariant mass distributions, shown in Figs. 1 and 2. To take advantage of the difference in the $R_{x y}$ distribution for background and signal, the data are divided into six $R_{x y}$ bins. The data are further split according to data taking year to account for differences in running conditions and Higgs boson production cross-section. The signal efficiency for each $R_{x y}$ bin is obtained from the simulated samples with $\pi_{\mathrm{v}}$ lifetimes of 10 and $100 \mathrm{ps}$, with the decay time dis-
Fig. 2 Dijet invariant mass distribution in the different $R_{x y}$ bins, for the 2012 data sample. For illustration, the best fit with a signal $\pi_{\mathrm{v}}$ model with mass $35 \mathrm{GeV} / \mathrm{c}^{2}$ and lifetime $10 \mathrm{ps}$ is overlaid. The solid blue line indicates the total background model, the short-dashed green line indicates the signal model for signal strength $\mu=1$, and the long-dashed red line indicates the best-fit signal strength
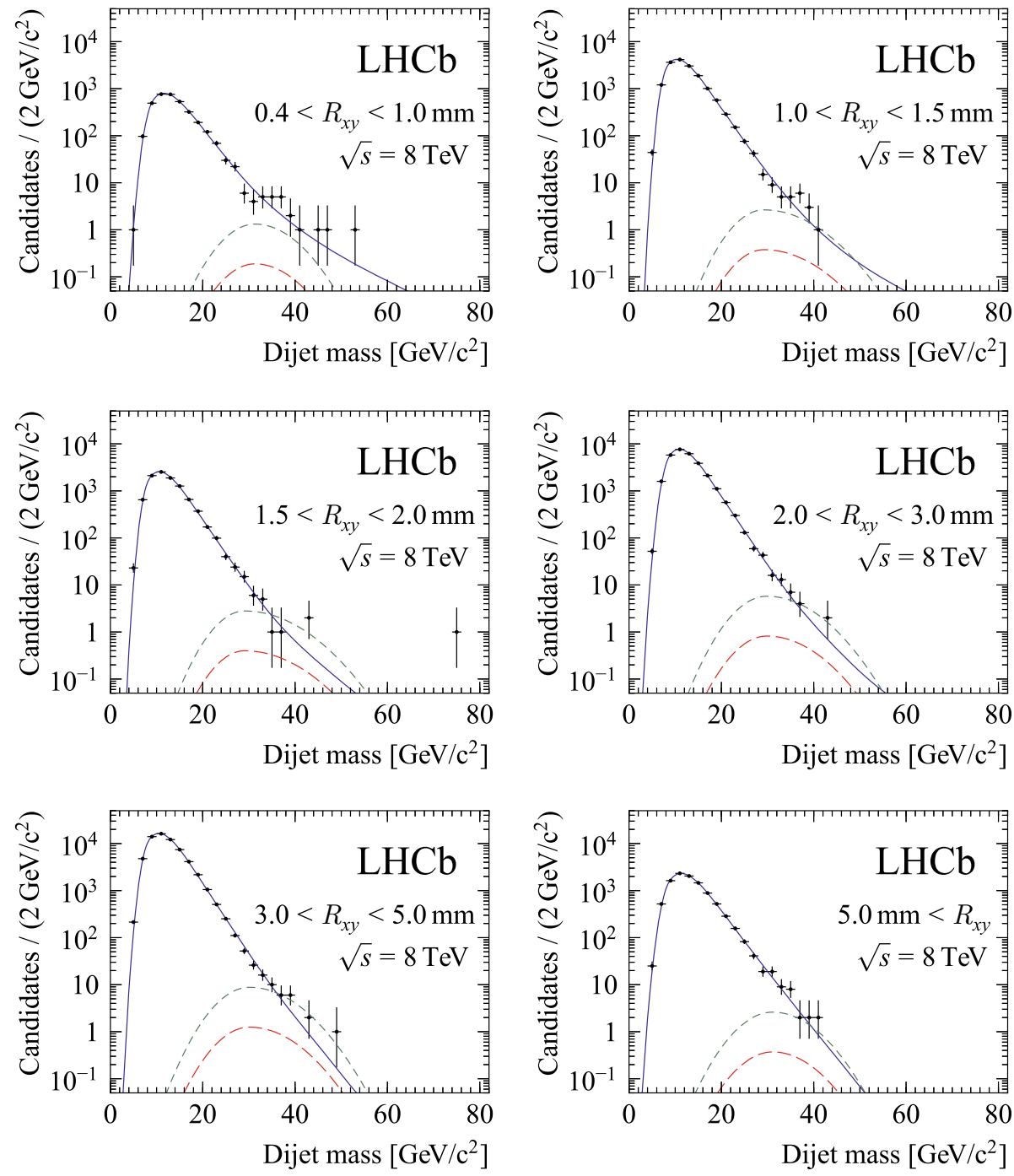
Fig. 3 Expected (open circles and dotted line) and observed (filled circles and solid line) upper limit versus lifetime for different $\pi_{\mathrm{v}}$ masses and decay modes. The green (dark) and yellow (light) bands indicate the quantiles of the expected upper limit corresponding to $\pm 1 \sigma$ and $\pm 2 \sigma$ for a Gaussian distribution. The decay $\pi_{\mathrm{v}} \rightarrow b \bar{b}$ is assumed, unless specified otherwise
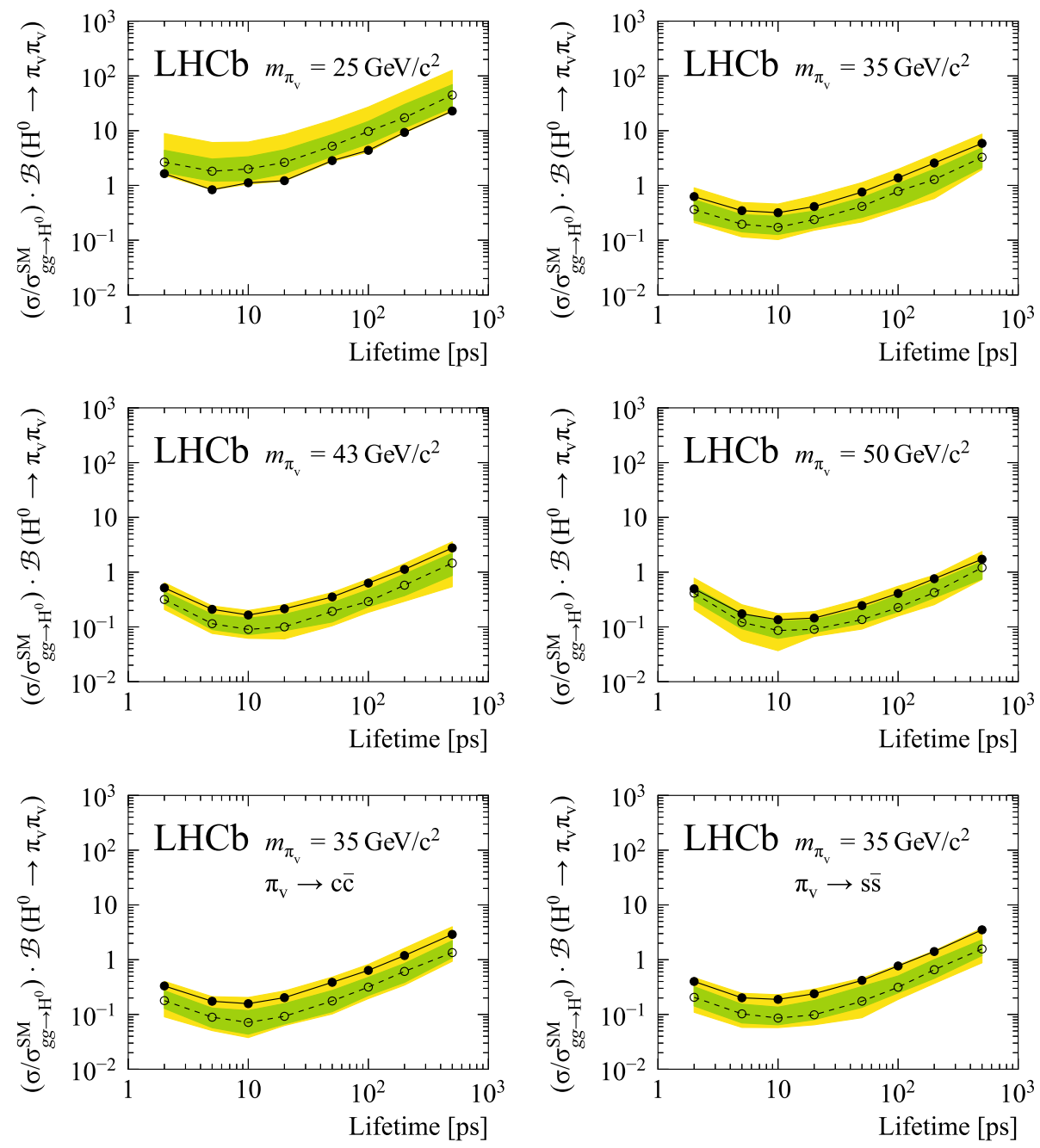

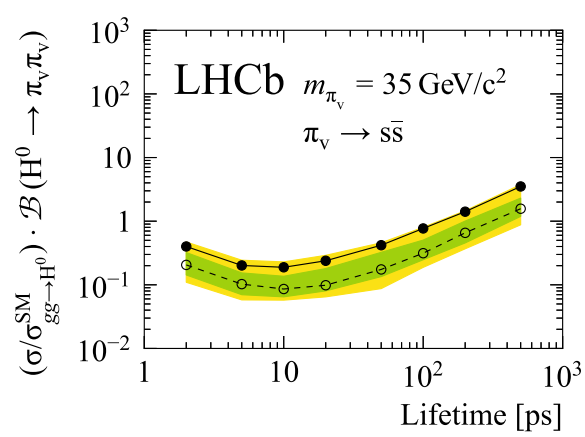

tributions reweighted to mimic other lifetime hypotheses as needed.

Results are presented as upper limits on the signal strength $\mu \equiv\left(\sigma / \sigma_{g g \rightarrow H^{0}}^{S M}\right) \cdot \mathcal{B}\left(H^{0} \rightarrow \pi_{\mathrm{v}} \pi_{\mathrm{v}}\right)$, where $\sigma$ is the excluded signal cross-section, $\sigma_{g g \rightarrow H^{0}}^{S M}$ is the SM Higgs boson production cross-section via the gluon fusion process and $\mathcal{B}\left(H^{0} \rightarrow \pi_{\mathrm{v}} \pi_{\mathrm{v}}\right)$ is the branching fraction of the Higgs boson decay to $\pi_{\mathrm{v}}$ particles. The branching fraction $\mathcal{B}_{q \bar{q}}$ of the $\pi_{\mathrm{v}}$ particle to the $q \bar{q}$ final state (with $q \bar{q}=b \bar{b}, c \bar{c}$ or $s \bar{s}$ depending on the final state under study) is assumed to be $100 \%$. If the decay width of the $\pi_{\mathrm{v}}$ particle is dominated by other decays than that under study, the limits scale as $1 /\left(\mathcal{B}_{q \bar{q}}\left(2-\mathcal{B}_{q \bar{q}}\right)\right)$. The Higgs boson production cross-section is assumed to be $15.11 \mathrm{pb}$ at $7 \mathrm{TeV}$ and $19.24 \mathrm{pb}$ at $8 \mathrm{TeV}$ [41].

The $\mathrm{CL}_{s}$ method [42] is used to determine upper limits. The profile likelihood ratio $q_{\mathrm{PLL}}^{\mu}=L(\mu, \hat{\theta}(\mu)) / L(\hat{\mu}, \hat{\theta})$ is chosen as a test statistic, where $L(\mu, \theta)$ denotes the likelihood as a function of $\mu$ and a set of nuisance parameters $\theta$, which are also extracted from the data; $L(\mu, \hat{\theta}(\mu))$ is the maximum likelihood for a hypothesized value of $\mu$ and $L(\hat{\mu}, \hat{\theta})$ is the global maximum likelihood. To estimate the sensitivity of the analysis and the significance of a potential signal, the expected upper limit quantiles in the case of zero signal are also evaluated.

For each value of $\mu$ and $\theta$ the likelihood is evaluated as $L(\mu, \theta)=\prod_{i} P\left(x_{i} ; \mu, \theta\right)$, where $P$ is the probability density for event $i$ and the product runs over all selected events. The observables $x_{i}$ for each candidate include the dijet mass, $R_{x y}$ bin and data taking year. For each $R_{x y}$ bin and data taking year, the invariant mass distribution is modelled by the sum of background and signal components. The distribution for the signal is modelled as a Gaussian distribution whose parameters are obtained from fully simulated signal events. For the background distribution an empirical model, outlined below, is adopted.

Background candidates can be categorized into two contributions. The first category is mostly due to the combination 
Table 4 Observed $95 \% \mathrm{CL}$ signal strength $(\mu)$ upper limits for different $\pi_{\mathrm{v}}$ models

\begin{tabular}{lllllllll}
\hline$\pi_{\mathrm{v}}$ mass & \multicolumn{7}{l}{$\pi_{\mathrm{v}}$ lifetime $(\mathrm{ps})$} & \\
\cline { 2 - 8 } & 2 & 5 & 10 & 20 & 50 & 100 & 200 & 500 \\
\hline $25 \mathrm{GeV} / c^{2}$ & 1.64 & 0.83 & 1.12 & 1.22 & 2.84 & 4.37 & 9.28 & 22.82 \\
$35 \mathrm{GeV} / c^{2}$ & 0.63 & 0.35 & 0.32 & 0.41 & 0.76 & 1.37 & 2.56 & 5.86 \\
$43 \mathrm{GeV} / c^{2}$ & 0.52 & 0.21 & 0.16 & 0.21 & 0.35 & 0.63 & 1.12 & 2.77 \\
$50 \mathrm{GeV} / c^{2}$ & 0.50 & 0.17 & 0.14 & 0.15 & 0.25 & 0.41 & 0.76 & 1.72 \\
$35 \mathrm{GeV} / c^{2}, \pi_{\mathrm{v}} \rightarrow c \bar{c}$ & 0.33 & 0.17 & 0.16 & 0.20 & 0.39 & 0.64 & 1.19 & 2.90 \\
$35 \mathrm{GeV} / c^{2}, \pi_{\mathrm{v}} \rightarrow s \bar{s}$ & 0.40 & 0.20 & 0.19 & 0.24 & 0.42 & 0.77 & 1.41 & 3.51 \\
\hline
\end{tabular}

of a heavy-flavour decay vertex or an interaction with detector material with particles from a primary interaction. This contribution has a steeply decreasing invariant mass spectrum. Following the approach in Ref. [9], the distribution is modelled by the convolution of a falling exponential distribution with a bifurcated Gaussian. All parameters of this background model are free to vary in the fit.

The second category is due to Standard Model dijet events. These events have candidates with jets that are approximately back-to-back in the transverse plane. It is suppressed by the selection on the dijet opening angle $\Delta R$. Its remaining contribution has a less steeply falling mass spectrum. It is described in the fit with a similar functional shape as for the first category, but with the parameters and the relative yields in the different bins fixed from a fit to the invariant mass distribution of candidates that fail the $\Delta R$ requirement. In the final fit only the total normalization of this component is varied. The second component is new compared to the model used for the previous analysis [9]. It leads to a better description of the high-mass tail, at the expense of one extra fit parameter for each data taking year. It was found that the result of the fit is not sensitive to the exact $\Delta R$ requirement used to select the events for this component.

All parameters of the fit to the invariant mass distribution are allowed to float independently in each bin, except for the following nuisance parameters: the dijet invariant mass scale, the overall signal efficiency, and the normalization for the second background contribution. All relevant systematic uncertainties are incorporated in the fit model: the overall uncertainty on the efficiency, as described in Sect. 4, the uncertainty on the dijet invariant mass scale, and the uncertainties on the shape parameters and relative normalisation arising from the finite size of the simulated signal samples. Gaussian constraints on these parameters are added to the likelihood.

Alternatives have been considered for the background mass model, in particular with an additional less steeply falling exponential to describe the tail. With these models the estimated background yield at higher mass is similar or

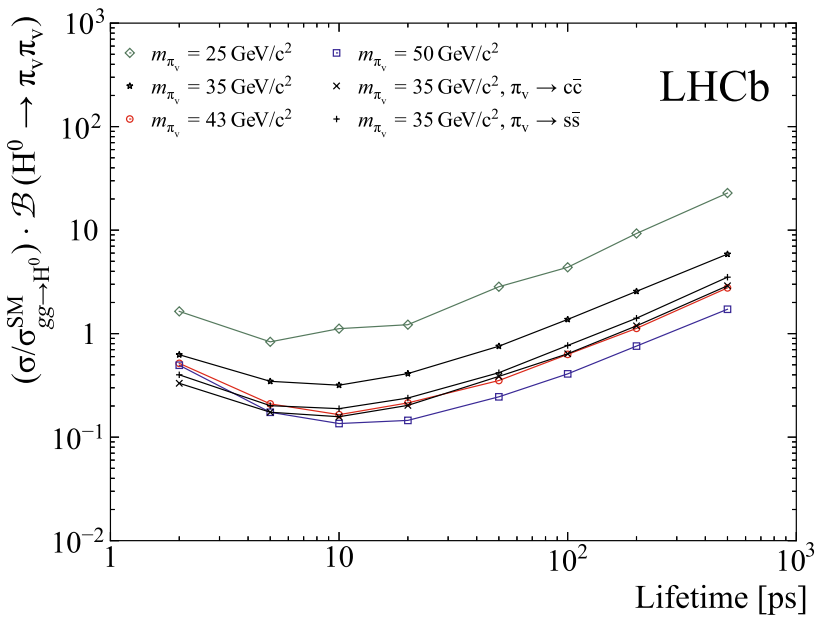

Fig. 4 Observed upper limit versus lifetime for different $\pi_{\mathrm{v}}$ masses and decay modes. The decay $\pi_{\mathrm{v}} \rightarrow b \bar{b}$ is assumed, unless specified otherwise

larger than with the nominal background model, leading to tighter limits on the signal. As the nominal model gives the most conservative limit, no additional systematic uncertainty is assigned for background modeling.

There is no significant excess of signal in the data. Upper limits at $95 \%$ confidence level (CL) as a function of lifetime for hidden-valley models with different $\pi_{\mathrm{v}}$ mass and decay mode are shown in Fig. 3 and summarized in Table 4 and Fig. 4. The best sensitivity is obtained for a mass of about $50 \mathrm{GeV} / \mathrm{c}^{2}$ and a lifetime of about $10 \mathrm{ps}$. The main improvements with respect to the previous result [9] are due to the enlarged data sample, the improved trigger selections, and the addition of the $R_{x y}$ bin above $5 \mathrm{~mm}$, which contributes to the increased sensitivity at larger lifetimes.

\section{Conclusion}

Results have been presented from a search for long-lived particles with a mass in the range $25-50 \mathrm{GeV} / c^{2}$ and a lifetime between 2 and 500 ps. The particles are assumed to be pair- 
produced in the decay of a $125 \mathrm{GeV} / c^{2}$ Standard-Model-like Higgs boson and to decay into two jets. Besides decays to $b \bar{b}$, which are the best motivated in the context of hidden-valley models $[1,2]$, also decays to $c \bar{c}$ and $s \bar{s}$ quark pairs are considered. No evidence for so far unknown long-lived particles is observed and limits are set as a function of mass and lifetime. These measurements complement other constraints on this production model at the LHC $[13,15]$ by placing stronger constraints at small masses and lifetimes.

Acknowledgements We express our gratitude to our colleagues in the CERN accelerator departments for the excellent performance of the LHC. We thank the technical and administrative staff at the LHCb institutes. We acknowledge support from CERN and from the national agencies: CAPES, CNPq, FAPERJ and FINEP (Brazil); MOST and NSFC (China); CNRS/IN2P3 (France); BMBF, DFG and MPG (Germany); INFN (Italy); NWO (The Netherlands); MNiSW and NCN (Poland); MEN/IFA (Romania); MinES and FASO (Russia); MinECo (Spain); SNSF and SER (Switzerland); NASU (Ukraine); STFC (United Kingdom); NSF (USA). We acknowledge the computing resources that are provided by CERN, IN2P3 (France), KIT and DESY (Germany), INFN (Italy), SURF (The Netherlands), PIC (Spain), GridPP (United Kingdom), RRCKI and Yandex LLC (Russia), CSCS (Switzerland), IFINHH (Romania), CBPF (Brazil), PL-GRID (Poland) and OSC (USA). We are indebted to the communities behind the multiple open source software packages on which we depend. Individual groups or members have received support from AvH Foundation (Germany), EPLANET, Marie Skłodowska-Curie Actions and ERC (European Union), Conseil Général de Haute-Savoie, Labex ENIGMASS and OCEVU, Région Auvergne (France), RFBR and Yandex LLC (Russia), GVA, XuntaGal and GENCAT (Spain), Herchel Smith Fund, The Royal Society, Royal Commission for the Exhibition of 1851 and the Leverhulme Trust (United Kingdom).

Open Access This article is distributed under the terms of the Creative Commons Attribution 4.0 International License (http://creativecomm ons.org/licenses/by/4.0/), which permits unrestricted use, distribution, and reproduction in any medium, provided you give appropriate credit to the original author(s) and the source, provide a link to the Creative Commons license, and indicate if changes were made.

Funded by SCOAP ${ }^{3}$.

\section{References}

1. M.J. Strassler, K.M. Zurek, Echoes of a hidden valley at hadron colliders. Phys. Lett. B 651, 374 (2007). arXiv:hep-ph/0604261

2. M.J. Strassler, K.M. Zurek, Discovering the Higgs through highly-displaced vertices. Phys. Lett. B 661, 263 (2008). arXiv:hep-ph/0605193

3. S. Chang, R. Dermisek, J.F. Gunion, N. Weiner, Nonstandard Higgs boson decays. Annu. Rev. Nucl. Part. Sci. 58, 75 (2008). arXiv:0801.4554

4. N. Craig, A. Katz, M. Strassler, R. Sundrum, Naturalness in the dark at the LHC. JHEP 07, 105 (2015). arXiv:1501.05310

5. D. Curtin, C.B. Verhaaren, Discovering uncolored naturalness in exotic Higgs decays. JHEP 12, 072 (2015). arXiv:1506.06141

6. ATLAS collaboration, G. Aad et al., Observation of a new particle in the search for the Standard Model Higgs boson with the ATLAS detector at the LHC. Phys. Lett. B 716, 1 (2012). arXiv:1207.7214
7. CMS collaboration, S. Chatrchyan et al., Observation of a new boson at a mass of $125 \mathrm{GeV}$ with the CMS experiment at the LHC. Phys. Lett. B 716, 30 (2012). arXiv: 1207.7235

8. ATLAS and CMS collaborations, G. Aad et al., Measurements of the Higgs boson production and decay rates and constraints on its couplings from a combined ATLAS and CMS analysis of the LHC pp collision data at $\sqrt{s}=7$ and 8 TeV. JHEP 08, 045 (2016). arXiv: 1606.02266

9. LHCb collaboration, R. Aaij et al., Search for long-lived particles decaying to jet pairs. Eur. Phys. J. C 75, 152 (2015). arXiv: 1412.3021

10. D0 collaboration, V.M. Abazov et al., Search for resonant pair production of long-lived particles decaying to $b \bar{b}$ in $p \bar{p}$ collisions at $\sqrt{s}=1.96$ TeV. Phys. Rev. Lett. 103, 071801 (2009). arXiv:0906.1787

11. CDF collaboration, T. Aaltonen et al., Search for heavy metastable particles decaying to jet pairs in pp collisions at $\sqrt{s}=1.96 \mathrm{TeV}$. Phys. Rev. D 85, 012007 (2012). arXiv: 1109.3136

12. ATLAS collaboration, G. Aad et al., Search for a light Higgs boson decaying to long-lived weakly-interacting particles in protonproton collisions at $\sqrt{s}=7 \mathrm{TeV}$ with the ATLAS detector. Phys. Rev. Lett. 108, 251801 (2012). arXiv:1203.1303

13. ATLAS collaboration, G. Aad et al., Search for long-lived, weakly interacting particles that decay to displaced hadronic jets in protonproton collisions at $\sqrt{s}=8 \mathrm{TeV}$ with the ATLAS detector. Phys. Rev. D 92, 012010 (2015). arXiv: 1504.03634

14. ATLAS collaboration, G. Aad et al., Search for pair-produced longlived neutral particles decaying in the ATLAS hadronic calorimeter in pp collisions at $\sqrt{s}=8 \mathrm{TeV}$. Phys. Lett. B 743, 15 (2015). arXiv: 1501.04020

15. CMS collaboration, V. Khachatryan et al., Search for long-lived neutral particles decaying to quark-antiquark pairs in protonproton collisions at $\sqrt{s}=8$ TeV. Phys. Rev. D 91, 012007 (2015). arXiv: 1411.6530

16. LHCb collaboration, R. Aaij et al., Search for Higgs-like bosons decaying into long-lived exotic particles. Eur. Phys. J. C 76, 664 (2016). arXiv: 1609.03124

17. LHCb collaboration, R. Aaij et al., Search for massive long-lived particles decaying semileptonically in the $\mathrm{LHCb}$ detector. Eur. Phys. J. C 77, 224 (2017). arXiv:1612.00945

18. LHCb collaboration, R. Aaij et al., Search for Majorana neutrinos in $B^{-} \rightarrow \pi^{+} \mu^{-} \mu^{-}$decays. Phys. Rev. Lett. 112, 131802 (2014). arXiv: 1401.5361

19. LHCb collaboration, R. Aaij et al., Searches for Majorana neutrinos in $B^{-}$decays. Phys. Rev. D 85, 112004 (2012). arXiv: 1201.5600

20. LHCb collaboration, R. Aaij et al., Search for hidden-sector bosons in $B^{0} \rightarrow K^{* 0} \chi\left(\mu^{+} \mu^{-}\right)$decays. Phys. Rev. Lett. 115, 161802 (2015). arXiv:1508.04094

21. LHCb collaboration, R. Aaij et al., Search for long-lived scalar particles in $B^{+} \rightarrow K^{+} \chi\left(\mu^{+} \mu^{-}\right)$decay. Phys. Rev. D 95, 071101 (2017). arXiv: 1612.07818

22. LHCb collaboration, A.A. Alves Jr. et al., The LHCb detector at the LHC. JINST 3, S08005 (2008)

23. LHCb collaboration, R. Aaij et al., LHCb detector performance. Int. J. Mod. Phys. A 30, 1530022 (2015). arXiv:1412.6352

24. T. Sjöstrand, S. Mrenna, P. Skands, A brief introduction to PYTHIA 8.1. Comput. Phys. Commun. 178, 852 (2008). arXiv:0710.3820

25. I. Belyaev et al., Handling of the generation of primary events in Gauss, the LHCb simulation framework. J. Phys. Conf. Ser. 331, 032047 (2011)

26. J. Pumplin et al., New generation of parton distributions with uncertainties from global QCD analysis. JHEP 07, 012 (2002). arXiv:hep-ph/0201195

27. Geant 4 collaboration, J. Allison et al., Geant 4 developments and applications. IEEE Trans. Nucl. Sci. 53, 270 (2006) 
28. Geant4 collaboration, S. Agostinelli et al., Geant4: a simulation toolkit. Nucl. Instrum. Methods A 506, 250 (2003)

29. M. Clemencic et al., The LHCb simulation application, Gauss: design, evolution and experience. J. Phys. Conf. Ser. 331, 032023 (2011)

30. R. Aaij et al., The LHCb trigger and its performance in 2011. JINST 8, P04022 (2013). arXiv: 1211.3055

31. M. Kucharczyk, P. Morawski, M. Witek, Primary vertex reconstruction at LHCb. LHCb-PUB-2014-044, Geneva: CERN

32. LHCb collaboration, R. Aaij et al., Study of forward Z+jet production in pp collisions at $\sqrt{s}=7$ TeV. JHEP 01, 033 (2014). arXiv: 1310.8197

33. M. Cacciari, G.P. Salam, G. Soyez, The anti- $k_{t}$ jet clustering algorithm. JHEP 04, 063 (2008). arXiv:0802.1189

34. SLD collaboration, $\mathrm{K}$. Abe et al., Measurement of $R_{b}$ using a vertex mass tag. Phys. Rev. Lett. 80, 660 (1998). arXiv:hep-ex/9708015

35. V. Heijne, Search for long-lived exotic particles at LHCb. PhD thesis (Vrije Universiteit, Amsterdam, 2016), CERN-THESIS-2014294
36. P.N.Y. David, Search for exotic long-lived particles with the $\mathrm{LHCb}$ detector. PhD thesis (Vrije Universiteit, Amsterdam, 2016), CERNTHESIS-2016-077

37. LHCb collaboration, R. Aaij et al., Measurement of the track reconstruction efficiency at LHCb. JINST 10, P02007 (2015). arXiv: 1408.1251

38. LHCb collaboration, R. Aaij et al., Prompt $K_{S}^{0}$ production in pp collisions at $\sqrt{s}=0.9$ TeV. Phys. Lett. B 693, 69 (2010). arXiv: 1008.3105

39. LHCb collaboration, R. Aaij et al., Measurement of $V^{0}$ production ratios in pp collisions at $\sqrt{s}=0.9$ and 7 TeV. JHEP 08, 034 (2011). arXiv: 1107.0882

40. LHCb collaboration, R. Aaij et al., Measurement of the $\mathrm{Z}+\mathrm{b}$-jet cross-section in pp collisions at $\sqrt{s}=7 \mathrm{TeV}$ in the forward region. JHEP 01, 064 (2015). arXiv:1411.1264

41. LHC Higgs Cross Section Working Group, S. Heinemeyer et al., Handbook of LHC Higgs cross sections: 3. Higgs properties. CERN-2013-004. arXiv:1307.1347

42. A.L. Read, Presentation of search results: the $C L_{S}$ technique. J. Phys. G 28, 2693 (2002)

\section{LHCb Collaboration}

R. Aaij ${ }^{40}$, B. Adeva ${ }^{39}$, M. Adinolfi ${ }^{48}$, Z. Ajaltouni ${ }^{5}$, S. Akar ${ }^{59}$, J. Albrecht ${ }^{10}$, F. Alessio ${ }^{40}$, M. Alexander ${ }^{53}$, S. Ali ${ }^{43}$, G. Alkhazov ${ }^{31}$, P. Alvarez Cartelle ${ }^{55}$, A. A. AlvesJr ${ }^{59}$, S. Amato ${ }^{2}$, S. Amerio ${ }^{23}$, Y. Amhis ${ }^{7}$, L. An ${ }^{3}$, L. Anderlini ${ }^{18}$, G. Andreassi ${ }^{41}$, M. Andreotti17,g, J. E. Andrews ${ }^{60}$, R. B. Appleby ${ }^{56}$, F. Archillii3 ${ }^{43}$, P. d'Argent ${ }^{12}$, J. Arnau Romeu ${ }^{6}$, A. Artamonov ${ }^{37}$, M. Artuso ${ }^{61}$, E. Aslanides ${ }^{6}$, G. Auriemma ${ }^{26}$, M. Baalouch ${ }^{5}$, I. Babuschkin ${ }^{56}$, S. Bachmann ${ }^{12}$, J. J. Back ${ }^{50}$, A. Badalov ${ }^{38}$, C. Baesso ${ }^{62}$, S. Baker ${ }^{55}$, V. Balagura ${ }^{7, c}$, W. Baldini ${ }^{17}$, A. Baranov ${ }^{35}$, R. J. Barlow ${ }^{56}$, C. Barschel ${ }^{40}$, S. Barsuk ${ }^{7}$, W. Barter ${ }^{56}$, F. Baryshnikov ${ }^{32}$, M. Baszczyk ${ }^{27,1}$, V. Batozskaya ${ }^{29}$, V. Battista ${ }^{41}$, A. Bay ${ }^{41}$, L. Beaucourt ${ }^{4}$, J. Beddow ${ }^{53}$, F. Bedeschi' ${ }^{24}$, I. Bediaga ${ }^{1}$, A. Beiter ${ }^{61}$, L. J. Bel ${ }^{43}$, V. Bellee ${ }^{41}$, N. Belloli ${ }^{21, i}$, K. Belous ${ }^{37}$, I. Belyaev ${ }^{32}$, E. Ben-Haim ${ }^{8}$, G. Bencivenni ${ }^{19}$, S. Benson ${ }^{43}$, S. Beranek ${ }^{9}$, A. Berezhnoy ${ }^{33}$, R. Bernet ${ }^{42}$, A. Bertolin ${ }^{23}$, C. Betancourt ${ }^{42}$, F. Betti ${ }^{15}$, M.-O. Bettler ${ }^{40}$, M. van Beuzekom ${ }^{43}$, Ia. Bezshyiko ${ }^{42}$, S. Bifani4 ${ }^{47}$, P. Billoir ${ }^{8}$, T. Bird ${ }^{56}$, A. Birnkraut ${ }^{10}$, A. Bitadze ${ }^{56}$, A. Bizzeti ${ }^{18, u}$, T. Blake ${ }^{50}$, F. Blanc ${ }^{41}$, J. Blouw ${ }^{11 \dagger}$, S. Blusk ${ }^{61}$, V. Bocci ${ }^{26}$, T. Boettcher ${ }^{58}$, A. Bondar ${ }^{36, w}$, N. Bondar ${ }^{31,40}$, W. Bonivento ${ }^{16}$, I. Bordyuzhin ${ }^{32}$, A. Borgheresi ${ }^{21, \mathrm{i}}$, S. Borghi ${ }^{56}$, M. Borisyak ${ }^{35}$, M. Borsato ${ }^{39}$, F. Bossu ${ }^{7}$, M. Boubdir ${ }^{9}$, T. J. V. Bowcock ${ }^{54}$, E. Bowen ${ }^{42}$, C. Bozzi ${ }^{17,40}$, S. Braun ${ }^{12}$, M. Britsch ${ }^{12}$, T. Britton ${ }^{61}$, J. Brodzicka ${ }^{56}$, E. Buchanan ${ }^{48}$, C. Burr ${ }^{56}$, A. Bursche ${ }^{16}$, J. Buytaert ${ }^{40}$, S. Cadeddu ${ }^{16}$, R. Calabrese ${ }^{17, g}$, M. Calvi ${ }^{21, i}$, M. Calvo Gomez ${ }^{38, m}$, A. Camboni ${ }^{38}$, P. Campana ${ }^{19}$, D. H. Campora Perez ${ }^{40}$, L. Capriotti ${ }^{56}$, A. Carbone ${ }^{15, \mathrm{e}}$, G. Carboni ${ }^{25, \mathrm{j}}$, R. Cardinale ${ }^{20, \mathrm{~h}}$, A. Cardini ${ }^{16}$, P. Carniti ${ }^{21, \mathrm{i}}$, L. Carson ${ }^{52}$, K. Carvalho Akiba ${ }^{2}$, G. Casse ${ }^{54}$,L. Cassina ${ }^{21, \mathrm{i}}$, L. Castillo Garcia ${ }^{41}$, M. Cattaneo ${ }^{40}$, G. Cavallero ${ }^{20, h}$, R. Cenci ${ }^{24, t}$, D. Chamont ${ }^{7}$, M. Charles $^{8}$, Ph. Charpentier ${ }^{40}$, G. Chatzikonstantinidis ${ }^{47}$, M. Chefdeville ${ }^{4}$, S. Chen $^{56}$, S.F. Cheung ${ }^{57}$ ， V. Chobanova ${ }^{39}$, M. Chrzaszcz ${ }^{27,42}$, X. Cid Vidal ${ }^{39}$, G. Ciezarek ${ }^{43}$, P. E. L. Clarke ${ }^{52}$, M. Clemencic ${ }^{40}$, H. V. Cliff ${ }^{49}$, J. Closier ${ }^{40}$, V. $\operatorname{Coco}^{59}$, J. Cogan $^{6}$, E. Cogneras ${ }^{5}$, V. Cogoni ${ }^{16,40, f}$, L. Cojocariu ${ }^{30}$, P. Collins ${ }^{40}$, A. Comerma-Montells ${ }^{12}$, A. Contu ${ }^{40}$, A. Cook $^{48}$, G. Coombs ${ }^{40}$, S. Coquereau ${ }^{38}$, G. Corti ${ }^{40}$, M. Corvo ${ }^{17, \mathrm{~g}}$, C. M. Costa Sobral ${ }^{50}$, B. Couturier ${ }^{40}$, G. A. Cowan ${ }^{52}$, D. C. Craik $^{52}$, A. Crocombe ${ }^{50}$, M. Cruz Torres ${ }^{62}$, S. Cunliffe ${ }^{55}$, R. Currie ${ }^{52}$, C. D'Ambrosio ${ }^{40}$, F. Da Cunha Marinho ${ }^{2}$, E. Dall'Occo ${ }^{43}$, J. Dalseno ${ }^{48}$, P. N. Y. David ${ }^{43}$ (1), A. Davis ${ }^{3}$, K. De Bruyn ${ }^{6}$, S. De Capua ${ }^{56}$, M. De Cian ${ }^{12}$ ， J. M. De Miranda ${ }^{1}$ ， L. De Paula ${ }^{2}$, M. De Serio ${ }^{14, d}$, P. De Simone ${ }^{19}$, C.T. Dean ${ }^{53}$, D. Decamp ${ }^{4}$, M. Deckenhoff ${ }^{10}$, L. Del Buono ${ }^{8}$, M. Demmer ${ }^{10}$, A. Dendek ${ }^{28}$, D. Derkach ${ }^{35}$, O. Deschamps ${ }^{5}$, F. Dettori ${ }^{40}$ ， B. Dey ${ }^{22}$, A. Di Canto ${ }^{40}$, H. Dijkstra ${ }^{40}$, F. Dordei ${ }^{40}$, M. Dorigo ${ }^{41}$, A. Dosil Suárez ${ }^{39}$, A. Dovbnya ${ }^{45}$, K. Dreimanis ${ }^{54}$ ， L. Dufour ${ }^{43}$ ，G. Dujany ${ }^{56}$, K. Dungs ${ }^{40}$, P. Durante ${ }^{40}$ ，R. Dzhelyadin ${ }^{37}$ ，A. Dziurda ${ }^{40}$, A. Dzyuba ${ }^{31}$, N. Déléage ${ }^{4}$,S. Easo $^{51}$, M. Ebert ${ }^{52}$, U. Egede ${ }^{55}$, V. Egorychev $^{32}$, S. Eidelman ${ }^{36, w}$, S. Eisenhardt ${ }^{52}$, U. Eitschberger ${ }^{10}$, R. Ekelhof ${ }^{10}$, L. Eklund ${ }^{53}$ ，S. Ely ${ }^{61}$, S. Esen ${ }^{12}$, H. M. Evans ${ }^{49}$, T. Evans ${ }^{57}$ ，A. Falabella ${ }^{15}$ ， N. Farley ${ }^{47}$, S. Farry ${ }^{54}$, R. Fay ${ }^{54}$ ，D. Fazzini ${ }^{21, i}$ ，D. Ferguson ${ }^{52}$, G. Fernandez ${ }^{38}$, A. Fernandez Prieto ${ }^{39}$, F. Ferrari ${ }^{15,40}$, F. Ferreira Rodrigues ${ }^{2}$, M. Ferro-Luzzi ${ }^{40}$, S. Filippov ${ }^{34}$, R. A. Fini ${ }^{14}$, M. Fiore ${ }^{17, g}$, M. Fiorini ${ }^{17, g}$, M. Firlej ${ }^{28}$, C. Fitzpatrick ${ }^{41}$, T. Fiutowski ${ }^{28}$, F. Fleuret ${ }^{7, b}$, K. Fohl ${ }^{40}$, M. Fontana ${ }^{16,40}$, F. Fontanelli20,h, D. C. Forshaw ${ }^{61}$, R. Forty ${ }^{40}$, V. Franco Lima ${ }^{54}$, M. Frank ${ }^{40}$, C. Frei $^{40}$, J. Fu ${ }^{22, q}$, W. Funk ${ }^{40}$, E. Furfaro ${ }^{25, j}$, C. Färber ${ }^{40}$, A. Gallas Torreira ${ }^{39}$, D. Galli ${ }^{15, \text { e }}$, S. Gallorini ${ }^{23}$, S. Gambetta ${ }^{52}$, M. Gandelman ${ }^{2}$, P. Gandini ${ }^{57}$, Y. Gao ${ }^{3}$, L. M. Garcia Martin ${ }^{69}$ ， J. García Pardiñas ${ }^{39}$ ， J. Garra Tico ${ }^{49}$ ， L. Garrido ${ }^{38}$, 
P. J. Garsed ${ }^{49}$ ，D. Gascon ${ }^{38}$ ，C. Gaspar ${ }^{40}$ ，L. Gavardi ${ }^{10}$ ，G. Gazzoni ${ }^{5}$ ，D. Gerick ${ }^{12}$ ，E. Gersabeck ${ }^{12}$ ， M. Gersabeck ${ }^{56}$, T. Gershon ${ }^{50}$, Ph. Ghez ${ }^{4}$ ，S. Giani ${ }^{41}$ ， V. Gibson ${ }^{49}$ ， O. G. Girard ${ }^{41}$ ， L. Giubega ${ }^{30}$ ， K. Gizdov ${ }^{52}$, V. V. Gligorov ${ }^{8}$, D. Golubkov ${ }^{32}$, A. Golutvin ${ }^{40,55}$, A. Gomes ${ }^{1, a}$, I. V. Gorelov ${ }^{33}$, C. Gotti²1,i, E. Govorkova ${ }^{43}$, R. Graciani Diaz ${ }^{38}$, L. A. Granado Cardoso ${ }^{40}$ ， E. Graugés ${ }^{38}$ ， E. Graverini ${ }^{42}, \quad$ G. Graziani ${ }^{18}, \quad$ A. Grecu ${ }^{30}, \quad$ R. Greim ${ }^{9}, \quad$ P. Griffith ${ }^{16}$, L. Grillo ${ }^{21,40, i}$, B. R. Gruberg Cazon ${ }^{57}$, O. Grünberg ${ }^{67}$, E. Gushchin ${ }^{34}$, Yu. Guz ${ }^{37}$, T. Gys ${ }^{40}$, C. Göbel ${ }^{62}$, T. Hadavizadeh ${ }^{57}$, C. Hadjivasiliou ${ }^{5}$ ， G. Haefeli ${ }^{41}$ ， C. Haen ${ }^{40}$ ， S. C. Haines ${ }^{49}$ ， B. Hamilton ${ }^{60}$ ， X. Han ${ }^{12}$ ， S. Hansmann-Menzemer ${ }^{12}$, N. Harnew ${ }^{57}$, S. T. Harnew ${ }^{48}$, J. Harrison ${ }^{56}$, M. Hatch ${ }^{40}$, J. He ${ }^{63}$, T. Head ${ }^{41}$, A. Heister ${ }^{9}$, K. Hennessy ${ }^{54}$, P. Henrard ${ }^{5}$, L. Henry ${ }^{8}$, E. van Herwijnen ${ }^{40}$, M. Heß ${ }^{67}$, A. Hicheur ${ }^{2}$, D. Hill ${ }^{57}$, C. Hombach ${ }^{56}$, P. H. Hopchev ${ }^{41}$, W. Hulsbergen ${ }^{43}$,

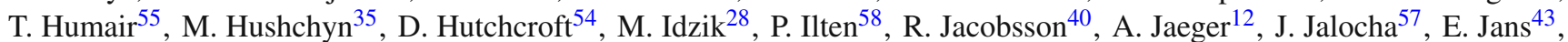
A. Jawahery ${ }^{60}$, F. Jiang ${ }^{3}$ ， M. John ${ }^{57}$ ，D. Johnson ${ }^{40}$, C. R. Jones ${ }^{49}$ ，C. Joram ${ }^{40}$ ， B. Jost ${ }^{40}$ ， N. Jurik ${ }^{57}$ ， S. Kandybei ${ }^{45}$, M. Karacson ${ }^{40}$, J. M. Kariuki ${ }^{48}$, S. Karodia ${ }^{53}$, M. Kecke ${ }^{12}$, M. Kelsey ${ }^{61}$, M. Kenzie ${ }^{49}$, T. Ketel ${ }^{44}$, E. Khairullin ${ }^{35}$,

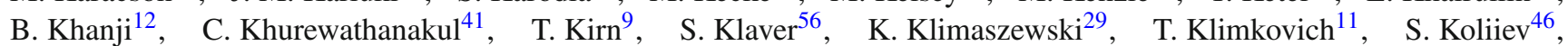
M. Kolpin ${ }^{12}$, I. Komarov ${ }^{41}$, P. Koppenburg ${ }^{43}$, A. Kosmyntseva ${ }^{32}$, M. Kozeiha ${ }^{5}$, L. Kravchuk ${ }^{34}$, K. Kreplin ${ }^{12}$, M. Kreps ${ }^{50}$, P. Krokovny ${ }^{36, w}$, F. Kruse ${ }^{10}$, W. Krzemien ${ }^{29}$, W. Kucewicz ${ }^{27,1}$, M. Kucharczyk ${ }^{27}$, V. Kudryavtsev ${ }^{36, w}$, A. K. Kuonen ${ }^{41}$, K. Kurek ${ }^{29}$, T. Kvaratskheliya ${ }^{32,40}$, D. Lacarrere ${ }^{40}$, G. Lafferty ${ }^{56}$, A. Lai ${ }^{16}$, G. Lanfranchi ${ }^{19}$, C. Langenbruch ${ }^{9}$, T. Latham ${ }^{50}$, C. Lazzeroni ${ }^{47}$, R. Le Gac ${ }^{6}$, J. van Leerdam ${ }^{43}$, A. Leflat ${ }^{33,40}$, J. Lefrançois ${ }^{7}$, R. Lefèvre ${ }^{5}$, F. Lemaitre ${ }^{40}$, E. Lemos Cid ${ }^{39}$, O. Leroy ${ }^{6}$, T. Lesiak ${ }^{27}$, B. Leverington ${ }^{12}$, T. $\mathrm{Li}^{3}, \mathrm{Y}_{\mathrm{Li}}{ }^{7}$, T. Likhomanenko ${ }^{35,68}$, R. Lindner ${ }^{40}$, C. Linn ${ }^{40}$, F. Lionetto ${ }^{42}$,

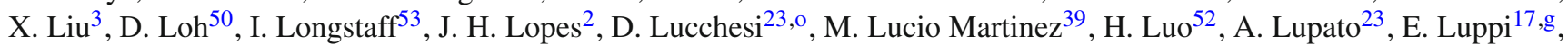
O. Lupton ${ }^{40}$, A. Lusiani24 ${ }^{24}$ X. Lyu ${ }^{63}$, F. Machefert ${ }^{7}$, F. Maciuc ${ }^{30}$, O. Maev ${ }^{31}$, K. Maguire ${ }^{56}$, S. Malde ${ }^{57}$, A. Malinin ${ }^{68}$, T. Maltsev ${ }^{36}$, G. Manca ${ }^{16, f}$, G. Mancinelli ${ }^{6}$, P. Manning ${ }^{61}$, J. Maratas ${ }^{5, v}$, J. F. Marchand ${ }^{4}$, U. Marconi ${ }^{15}$, C. Marin Benito ${ }^{38}$, M. Marinangeli ${ }^{41}$, P. Marino ${ }^{24, t}$, J. Marks ${ }^{12}$, G. Martellotti ${ }^{26}$, M. Martin ${ }^{6}$, M. Martinelli ${ }^{41}$, D. Martinez Santos ${ }^{39}$, F. Martinez Vidal ${ }^{69}$, D. Martins Tostes ${ }^{2}$, L. M. Massacrier ${ }^{7}$, A. Massafferri ${ }^{1}$, R. Matev ${ }^{40}$, A. Mathad ${ }^{50}$, Z. Mathe ${ }^{40}$, C. Matteuzzi ${ }^{21}$, A. Mauri ${ }^{42}$, E. Maurice ${ }^{7, b}$, B. Maurin ${ }^{41}$, A. Mazurov $^{47}$, M. McCann ${ }^{55,40}$, A. McNab ${ }^{56}$, R. McNulty ${ }^{13}$, B. Meadows ${ }^{59}$, F. Meier ${ }^{10}$, M. Meissner ${ }^{12}$, D. Melnychuk ${ }^{29}$, M. Merk ${ }^{43}$, A. Merli22,q, E. Michielin ${ }^{23}$, D. A. Milanes ${ }^{66}$, M.-N. Minard ${ }^{4}$, D. S. Mitzel ${ }^{12}$, A. Mogini ${ }^{8}$, J. Molina Rodriguez ${ }^{1}$, I. A. Monroy ${ }^{66}$, S. Monteil ${ }^{5}$, M. Morandinn ${ }^{23}$, P. Morawski ${ }^{28}$, A. Mordà ${ }^{6}$, M. J. Morello ${ }^{24, t}$, O. Morgunova ${ }^{68}$, J. Moron ${ }^{28}$, A. B. Morris ${ }^{52}$, R. Mountain ${ }^{61}$, F. Muheim ${ }^{52}$, M. Mulder ${ }^{43}$, M. Mussini ${ }^{15}$, D. Müller ${ }^{56}$, J. Müller ${ }^{10}$, K. Müller ${ }^{42}$, V. Müller ${ }^{10}$, P. Naik ${ }^{48}$, T. Nakada ${ }^{41}$, R. Nandakumar ${ }^{51}$, A. Nandi ${ }^{57}$, I. Nasteva ${ }^{2}$, M. Needham ${ }^{52}$, N. Neri' ${ }^{22}, \quad$ S. Neubert ${ }^{12}, \quad$ N. Neufeld ${ }^{40}, \quad$ M. Neuner ${ }^{12}, \quad$ T. D. Nguyen ${ }^{41}$, C. Nguyen-Mau ${ }^{41, \mathrm{n}}$, S. Nieswand ${ }^{9}$, R. Niet ${ }^{10}$, N. Nikitin ${ }^{33}$, T. Nikodem ${ }^{12}$, A. Nogay ${ }^{68}$, A. Novoselov ${ }^{37}$, D. P. O'Hanlon ${ }^{50}$, A. Oblakowska-Mucha ${ }^{28}$, V. Obraztsov ${ }^{37}$, S. Ogilvy ${ }^{19}$, R. Oldeman ${ }^{16, f}$, C. J. G. Onderwater ${ }^{70}$, J. M. Otalora Goicochea ${ }^{2}$, A. Otto ${ }^{40}$, P. Owen ${ }^{42}$, A. Oyanguren ${ }^{69}$, P. R. Pais ${ }^{41}$, A. Palano ${ }^{14, d}$, M. Palutan ${ }^{19}$, A. Papanestis ${ }^{51}$, M. Pappagallo ${ }^{14, d}$, L. L. Pappalardo ${ }^{17, \mathrm{~g}}$, W. Parker $^{60}$, C. Parkes ${ }^{56}$, G. Passaleva ${ }^{18}$, A. Pastore ${ }^{14, \mathrm{~d}}$, G. D. Patel ${ }^{54}$, M. Patel ${ }^{55}$, C. Patrignani ${ }^{15, \mathrm{e}}$, A. Pearce $^{40}$, A. Pellegrino ${ }^{43}$, G. Penso ${ }^{26}$, M. Pepe Altarelli ${ }^{40}$, S. Perazzini ${ }^{40}$, P. Perret ${ }^{5}$, L. Pescatore ${ }^{41}$, K. Petridis ${ }^{48}$, A. Petrolini ${ }^{20, h}$, A. Petrov ${ }^{68}$, M. Petruzzo ${ }^{22, q}$, E. Picatoste Olloqui ${ }^{38}$, B. Pietrzyk ${ }^{4}$, M. Pikies ${ }^{27}$, D. Pinci ${ }^{26}$, A. Pistone ${ }^{20, h}$, A. Piucci ${ }^{12}$, V. Placinta ${ }^{30}$, S. Playfer ${ }^{52}$, M. Plo Casasus ${ }^{39}$, T. Poikela ${ }^{40}$, F. Polci ${ }^{8}$, A. Poluektov ${ }^{36,50}$, I. Polyakov ${ }^{61}$, E. Polycarpo ${ }^{2}$, G. J. Pomery ${ }^{48}$, S. Ponce ${ }^{40}$, A. Popov ${ }^{37}$, D. Popov ${ }^{11,40}$, B. Popovicicio, S. Poslavskii ${ }^{37}$, C. Potterat ${ }^{2}$, E. Price ${ }^{48}$, J. D. Price ${ }^{54}$, J. Prisciandaro ${ }^{39}$, A. Pritchard ${ }^{54}$, C. Prouve ${ }^{48}$, V. Pugatch ${ }^{46}$, A. Puig Navarro ${ }^{42}$, G. Punzi ${ }^{24, p}$, W. Qian ${ }^{50}$, R. Quagliani ${ }^{7,48}$, B. Rachwal27, J. H. Rademacker ${ }^{48}$, M. Rama ${ }^{24}$, M. Ramos Pernas ${ }^{39}$, M. S. Rangel ${ }^{2}$, I. Raniuk ${ }^{45}$, F. Ratnikov ${ }^{35}$, G. Raven ${ }^{44}$, F. Redi ${ }^{55}$, S. Reichert ${ }^{10}$, A. C. dos Reis ${ }^{1}$, C. Remon Alepuz ${ }^{69}$, V. Renaudin ${ }^{7}$, S. Ricciardi ${ }^{51}$, S. Richards ${ }^{48}$, M. Rihl ${ }^{40}$, K. Rinnert ${ }^{54}$, V. Rives Molina ${ }^{38}$, P. Robbe ${ }^{7,40}$, A. B. Rodrigues ${ }^{1}$, E. Rodrigues ${ }^{59}$, J. A. Rodriguez Lopez ${ }^{66}$, P. Rodriguez Perez ${ }^{56 \dagger}$, A. Rogozhnikov ${ }^{35}$, S. Roiser ${ }^{40}$, A. Rollings ${ }^{57}$, V. Romanovskiy ${ }^{37}$, A. Romero Vidal ${ }^{39}$, J. W. Ronayne ${ }^{13}$, M. Rotondo ${ }^{19}$, M. S. Rudolph ${ }^{61}$, T. Ruf ${ }^{40}$, P. Ruiz Valls ${ }^{69}$, J. J. Saborido Silva ${ }^{39}$, E. Sadykhov ${ }^{32}$, N. Sagidova ${ }^{31}$, B. Saitta ${ }^{16, f}$, V. Salustino Guimaraes ${ }^{1}$, D. Sanchez Gonzalo ${ }^{38}$, C. Sanchez Mayordomo ${ }^{69}$,

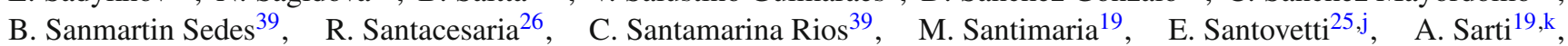
C. Satriano ${ }^{26, s}$, A. Satta ${ }^{25}$, D. M. Saunders ${ }^{48}$, D. Savrina ${ }^{32,33}$, S. Schael ${ }^{9}$, M. Schellenberg ${ }^{10}$, M. Schiller ${ }^{53}$, H. Schindler ${ }^{40}$, M. Schlupp ${ }^{10}$, M. Schmelling ${ }^{11}$, T. Schmelzer ${ }^{10}$, B. Schmidt ${ }^{40}$, O. Schneider ${ }^{41}$, A. Schopper ${ }^{40}$, H. F. Schreiner ${ }^{59}$, K. Schubert ${ }^{10}$, M. Schubiger ${ }^{41}$, M.-H. Schune ${ }^{7}$, R. Schwemmer ${ }^{40}$, B. Sciascia ${ }^{19}$, A. Sciubba ${ }^{26, k}$, A. Semennikov ${ }^{32}$, A. Sergi ${ }^{47}$, N. Serra ${ }^{42}$, J. Serrano ${ }^{6}$, L. Sestini ${ }^{23}$, P. Seyfert ${ }^{21}$, M. Shapkin ${ }^{37}$, I. Shapoval ${ }^{45}$, Y. Shcheglov ${ }^{31}$, T. Shears ${ }^{54}$, L. Shekhtman ${ }^{36, w}$, V. Shevchenko ${ }^{68}$, B. G. Siddi ${ }^{17,40}$, R. Silva Coutinho ${ }^{42}$, L. Silva de Oliveira ${ }^{2}$, G. Simi $^{23,0}$, S. Simone ${ }^{14, d}$, M. Sirendi ${ }^{49}$ ， N. Skidmore ${ }^{48}$ ， T. Skwarnicki ${ }^{61}$ ，E. Smith ${ }^{55}$ ， I. T. Smith ${ }^{52}$ ， J. Smith ${ }^{49}$ ， M. Smith ${ }^{55}$ ， 1. Soares Lavra ${ }^{1}$, M. D. Sokoloff ${ }^{59}$, F. J. P. Soler ${ }^{53}$, B. Souza De Paula $^{2}$, B. Spaan $^{10}$, P. Spradlin ${ }^{53}$, S. Sridharan ${ }^{40}$, F. Stagni ${ }^{40}$, M. Stahl ${ }^{12}$, S. Stahl ${ }^{40}$, P. Stefko ${ }^{41}$, S. Stefkova ${ }^{55}$, O. Steinkamp ${ }^{42}$, S. Stemmle ${ }^{12}$, O. Stenyakin ${ }^{37}$, H. Stevens ${ }^{10}$, S. Stevenson ${ }^{57}$, S. Stoica ${ }^{30}$, S. Stone ${ }^{61}$, B. Storaci ${ }^{42}$, S. Stracka ${ }^{24, p}$, M. E. Stramaglia ${ }^{41}$, M. Straticiuc ${ }^{30}$, U. Straumann ${ }^{42}$, L. Sun ${ }^{64}$, 
W. Sutcliffe ${ }^{55}$, K. Swientek ${ }^{28}$, V. Syropoulos ${ }^{44}$, M. Szczekowski ${ }^{29}$, T. Szumlak ${ }^{28}$, S. T'Jampens ${ }^{4}$, A. Tayduganov ${ }^{6}$, T. Tekampe ${ }^{10}$, G. Tellarini ${ }^{17, g}$, F. Teubert ${ }^{40}$, E. Thomas ${ }^{40}$, J. van Tilburg ${ }^{43}$, M. J. Tilley ${ }^{55}$, V. Tisserand ${ }^{4}$, M. Tobin ${ }^{41}$, S. Tolk ${ }^{49}$, L. Tomassetti ${ }^{17, g}$, D. Tonelli ${ }^{40}$, S. Topp-Joergensen ${ }^{57}$, F. Toriello ${ }^{61}$, E. Tournefier ${ }^{4}$, S. Tourneur $^{41}$, K. Trabelsi $^{41}$, M. Traill ${ }^{53}$, M. T. $\operatorname{Tran}^{41}$, M. Tresch ${ }^{42}$, A. Trisovic ${ }^{40}$, A. Tsaregorodtsev6 ${ }^{6}$, P. Tsopelas ${ }^{43}$, A. Tully ${ }^{49}$, N. Tuning ${ }^{43}$, A. Ukleja ${ }^{29}$, A. Ustyuzhanin ${ }^{35}$, U. Uwer ${ }^{12}$, C. Vacca ${ }^{16, f}$, V. Vagnoni ${ }^{15,40}$, A. Valassi ${ }^{40}, \mathrm{~S}$. Valat ${ }^{40}$, G. Valenti ${ }^{15}$, R. Vazquez Gomez ${ }^{19}$, P. Vazquez Regueiro ${ }^{39}$, S. Vecchi ${ }^{17}$, M. van Veghel ${ }^{43}$, J. J. Velthuis ${ }^{48}$, M. Veltri ${ }^{18, r}$, G. Veneziano ${ }^{57}$, A. Venkateswaran ${ }^{61}$, M. Vernet ${ }^{5}$, M. Vesterinen ${ }^{12}$, J. V. Viana Barbosa ${ }^{40}$, B. Viaud ${ }^{7}$, D. Vieira ${ }^{63}$, M. Vieites Diaz ${ }^{39}$, H. Viemann ${ }^{67}$ X. Vilasis-Cardona ${ }^{38, m}$, M. Vitti ${ }^{49}$, V. Volkov ${ }^{33}$, A. Vollhardt ${ }^{42}$, B. Voneki ${ }^{40}$, A. Vorobyev ${ }^{31}$, V. Vorobyev ${ }^{36, w}$, C. Voß ${ }^{9}$, J. A. de Vries ${ }^{43}$, C. Vázquez Sierra ${ }^{39}$, R. Waldi ${ }^{67}$, C. Wallace ${ }^{50}$, R. Wallace ${ }^{13}$, J. Walsh ${ }^{24}$, J. Wang ${ }^{61}$, D. R. Ward ${ }^{49}$, H. M. Wark ${ }^{54}$, N. K. Watson ${ }^{47}$, D. Websdale ${ }^{55}$, A. Weiden ${ }^{42}$, M. Whitehead ${ }^{40}$, J. Wicht ${ }^{50}$, G. Wilkinson ${ }^{40,57}$, M. Wilkinson ${ }^{61}$, M. Williams ${ }^{40}$, M. P. Williams ${ }^{47}$, M. Williams ${ }^{58}$, T. Williams ${ }^{47}$, F. F. Wilson ${ }^{51}$, J. Wimberley ${ }^{60}$, M. A. Winn ${ }^{7}$, J. Wishahi ${ }^{10}$, W. Wislicki ${ }^{29}$, M. Witek ${ }^{27}$, G. Wormser ${ }^{7}$, S. A. Wotton ${ }^{49}$, K. Wraight ${ }^{53}$, K. Wyllie ${ }^{40}$, Y. Xie ${ }^{65}$, Z. Xu ${ }^{4}$, Z. Yang ${ }^{3}$, Y. Yao ${ }^{61}$, H. Yin ${ }^{65}$, J. Yu ${ }^{65}$, X. Yuan ${ }^{36, w}$, O. Yushchenko ${ }^{37}$, K. A. Zarebski ${ }^{47}$, M. Zavertyaev ${ }^{11, c}$, L. Zhang ${ }^{3}$, Y. Zhang ${ }^{7}$, A. Zhelezov ${ }^{12}$, Y. Zheng ${ }^{63}$, X. Zhu ${ }^{3}$, V. Zhukov ${ }^{33}$, S. Zucchelli ${ }^{15}$

${ }^{1}$ Centro Brasileiro de Pesquisas Físicas (CBPF), Rio de Janeiro, Brazil

${ }^{2}$ Universidade Federal do Rio de Janeiro (UFRJ), Rio de Janeiro, Brazil

${ }^{3}$ Center for High Energy Physics, Tsinghua University, Beijing, China

${ }^{4}$ LAPP, Université Savoie Mont-Blanc, CNRS/IN2P3, Annecy-Le-Vieux, France

${ }^{5}$ Clermont Université, Université Blaise Pascal, CNRS/IN2P3, LPC, Clermont-Ferrand, France

${ }^{6}$ CPPM, Aix-Marseille Université, CNRS/IN2P3, Marseille, France

${ }^{7}$ LAL, Université Paris-Sud, CNRS/IN2P3, Orsay, France

${ }^{8}$ LPNHE, Université Pierre et Marie Curie, Université Paris Diderot, CNRS/IN2P3, Paris, France

${ }^{9}$ I. Physikalisches Institut, RWTH Aachen University, Aachen, Germany

${ }^{10}$ Fakultät Physik, Technische Universität Dortmund, Dortmund, Germany

11 Max-Planck-Institut für Kernphysik (MPIK), Heidelberg, Germany

12 Physikalisches Institut, Ruprecht-Karls-Universität Heidelberg, Heidelberg, Germany

13 School of Physics, University College Dublin, Dublin, Ireland

14 Sezione INFN di Bari, Bari, Italy

15 Sezione INFN di Bologna, Bologna, Italy

16 Sezione INFN di Cagliari, Cagliari, Italy

${ }^{17}$ Universita e INFN Ferrara, Ferrara, Italy

18 Sezione INFN di Firenze, Florence, Italy

${ }^{19}$ Laboratori Nazionali dell'INFN di Frascati, Frascati, Italy

20 Sezione INFN di Genova, Genoa, Italy

${ }^{21}$ Universita \& INFN Milano Bicocca, Milan, Italy

22 Sezione di Milano, Milan, Italy

23 Sezione INFN di Padova, Padova, Italy

24 Sezione INFN di Pisa, Pisa, Italy

25 Sezione INFN di Roma Tor Vergata, Rome, Italy

26 Sezione INFN di Roma La Sapienza, Rome, Italy

${ }^{27}$ Henryk Niewodniczanski Institute of Nuclear Physics Polish Academy of Sciences, Kraków, Poland

${ }^{28}$ Faculty of Physics and Applied Computer Science, AGH-University of Science and Technology, Kraków, Poland

${ }^{29}$ National Center for Nuclear Research (NCBJ), Warsaw, Poland

${ }^{30}$ Horia Hulubei National Institute of Physics and Nuclear Engineering, Bucharest-Magurele, Romania

31 Petersburg Nuclear Physics Institute (PNPI), Gatchina, Russia

32 Institute of Theoretical and Experimental Physics (ITEP), Moscow, Russia

33 Institute of Nuclear Physics, Moscow State University (SINP MSU), Moscow, Russia

${ }^{34}$ Institute for Nuclear Research of the Russian Academy of Sciences (INR RAN), Moscow, Russia

35 Yandex School of Data Analysis, Moscow, Russia

${ }^{36}$ Budker Institute of Nuclear Physics (SB RAS), Novosibirsk, Russia

${ }^{37}$ Institute for High Energy Physics (IHEP), Protvino, Russia

${ }^{38}$ ICCUB, Universitat de Barcelona, Barcelona, Spain 
${ }^{39}$ Universidad de Santiago de Compostela, Santiago de Compostela, Spain

${ }^{40}$ European Organization for Nuclear Research (CERN), Geneva, Switzerland

${ }^{41}$ Institute of Physics, Ecole Polytechnique Fédérale de Lausanne (EPFL), Lausanne, Switzerland

${ }^{42}$ Physik-Institut, Universität Zürich, Zürich, Switzerland

${ }^{43}$ Nikhef National Institute for Subatomic Physics, Amsterdam, The Netherlands

${ }^{44}$ Nikhef National Institute for Subatomic Physics and VU University Amsterdam, Amsterdam, The Netherlands

${ }^{45}$ NSC Kharkiv Institute of Physics and Technology (NSC KIPT), Kharkiv, Ukraine

${ }^{46}$ Institute for Nuclear Research of the National Academy of Sciences (KINR), Kyiv, Ukraine

${ }^{47}$ University of Birmingham, Birmingham, UK

${ }^{48}$ H.H. Wills Physics Laboratory, University of Bristol, Bristol, UK

${ }^{49}$ Cavendish Laboratory, University of Cambridge, Cambridge, UK

${ }^{50}$ Department of Physics, University of Warwick, Coventry, UK

${ }^{51}$ STFC Rutherford Appleton Laboratory, Didcot, UK

${ }^{52}$ School of Physics and Astronomy, University of Edinburgh, Edinburgh, UK

${ }^{53}$ School of Physics and Astronomy, University of Glasgow, Glasgow, UK

${ }^{54}$ Oliver Lodge Laboratory, University of Liverpool, Liverpool, UK

55 Imperial College London, London, UK

${ }^{56}$ School of Physics and Astronomy, University of Manchester, Manchester, UK

${ }^{57}$ Department of Physics, University of Oxford, Oxford, UK

${ }^{58}$ Massachusetts Institute of Technology, Cambridge, MA, USA

${ }^{59}$ University of Cincinnati, Cincinnati, OH, USA

${ }^{60}$ University of Maryland, College Park, MD, USA

${ }^{61}$ Syracuse University, Syracuse, NY, USA

${ }^{62}$ Pontifícia Universidade Católica do Rio de Janeiro (PUC-Rio), Rio de Janeiro, Brazil, associated to ${ }^{2}$

${ }^{63}$ University of Chinese Academy of Sciences, Beijing, China, associated to ${ }^{3}$

${ }^{64}$ School of Physics and Technology, Wuhan University, Wuhan, China, associated to ${ }^{3}$

${ }^{65}$ Institute of Particle Physics, Central China Normal University, Wuhan, Hubei, China associated to ${ }^{3}$

${ }^{66}$ Departamento de Fisica, Universidad Nacional de Colombia, Bogotá, Colombia, associated to ${ }^{8}$

${ }^{67}$ Institut für Physik, Universität Rostock, Rostock, Germany, associated to ${ }^{12}$

${ }^{68}$ National Research Centre Kurchatov Institute, Moscow, Russia, associated to ${ }^{32}$

${ }^{69}$ Instituto de Fisica Corpuscular, Centro Mixto Universidad de Valencia-CSIC, Valencia, Spain, associated to ${ }^{38}$

${ }^{70}$ Van Swinderen Institute, University of Groningen, Groningen, The Netherlands, associated to ${ }^{43}$

${ }^{\text {a }}$ Universidade Federal do Triângulo Mineiro (UFTM), Uberaba-MG, Brazil

${ }^{\mathrm{b}}$ Laboratoire Leprince-Ringuet, Palaiseau, France

${ }^{c}$ P.N. Lebedev Physical Institute, Russian Academy of Science (LPI RAS), Moscow, Russia

d Università di Bari, Bari, Italy

${ }^{\mathrm{e}}$ Università di Bologna, Bologna, Italy

${ }^{\mathrm{f}}$ Università di Cagliari, Cagliari, Italy

g Università di Ferrara, Ferrara, Italy

${ }^{\mathrm{h}}$ Università di Genova, Genoa, Italy

${ }^{\text {i }}$ Università di Milano Bicocca, Milan, Italy

${ }^{\mathrm{j}}$ Università di Roma Tor Vergata, Rome, Italy

${ }^{\mathrm{k}}$ Università di Roma La Sapienza, Rome, Italy

${ }^{1}$ AGH-University of Science and Technology, Faculty of Computer Science, Electronics and Telecommunications, Kraków, Poland

${ }^{m}$ LIFAELS, La Salle, Universitat Ramon Llull, Barcelona, Spain

${ }^{\mathrm{n}}$ Hanoi University of Science, Hanoi, Vietnam

${ }^{\circ}$ Università di Padova, Padova, Italy

$\mathrm{p}$ Università di Pisa, Pisa, Italy

q Università degli Studi di Milano, Milan, Italy

${ }^{\mathrm{r}}$ Università di Urbino, Urbino, Italy

${ }^{\mathrm{s}}$ Università della Basilicata, Potenza, Italy 
t Scuola Normale Superiore, Pisa, Italy

u Università di Modena e Reggio Emilia, Modena, Italy

${ }^{v}$ Iligan Institute of Technology (IIT), Iligan, Philippines

w Novosibirsk State University, Novosibirsk, Russia

$\dagger$ Deceased 